\title{
Polarizer design for millimeter-wave plasma diagnostics
}

Leipold, Frank; Salewski, Mirko; Jacobsen, Asger Schou; Jessen, Martin; Korsholm, Søren Bang; Michelsen, Poul; Nielsen, Stefan Kragh; Stejner Pedersen, Morten

Published in:

Review of Scientific Instruments

Link to article, DOI:

$10.1063 / 1.4816724$

Publication date:

2013

Document Version

Publisher's PDF, also known as Version of record

Link back to DTU Orbit

Citation (APA):

Leipold, F., Salewski, M., Jacobsen, A. S., Jessen, M., Korsholm, S. B., Michelsen, P., Nielsen, S. K., \& Stejner Pedersen, M. (2013). Polarizer design for millimeter-wave plasma diagnostics. Review of Scientific Instruments, 84(8), 084701. https://doi.org/10.1063/1.4816724

\section{General rights}

Copyright and moral rights for the publications made accessible in the public portal are retained by the authors and/or other copyright owners and it is a condition of accessing publications that users recognise and abide by the legal requirements associated with these rights.

- Users may download and print one copy of any publication from the public portal for the purpose of private study or research.

- You may not further distribute the material or use it for any profit-making activity or commercial gain

- You may freely distribute the URL identifying the publication in the public portal

If you believe that this document breaches copyright please contact us providing details, and we will remove access to the work immediately and investigate your claim. 


\section{AIP Scientific Instruments}

\section{Polarizer design for millimeter-wave plasma diagnostics}

F. Leipold, M. Salewski, A. S. Jacobsen, M. Jessen, S. B. Korsholm et al.

Citation: Rev. Sci. Instrum. 84, 084701 (2013); doi: 10.1063/1.4816724

View online: http://dx.doi.org/10.1063/1.4816724

View Table of Contents: http://rsi.aip.org/resource/1/RSINAK/v84/i8

Published by the AIP Publishing LLC.

\section{Additional information on Rev. Sci. Instrum.}

Journal Homepage: http://rsi.aip.org

Journal Information: http://rsi.aip.org/about/about_the_journal

Top downloads: http://rsi.aip.org/features/most_downloaded

Information for Authors: http://rsi.aip.org/authors

\section{ADVERTISEMENT}

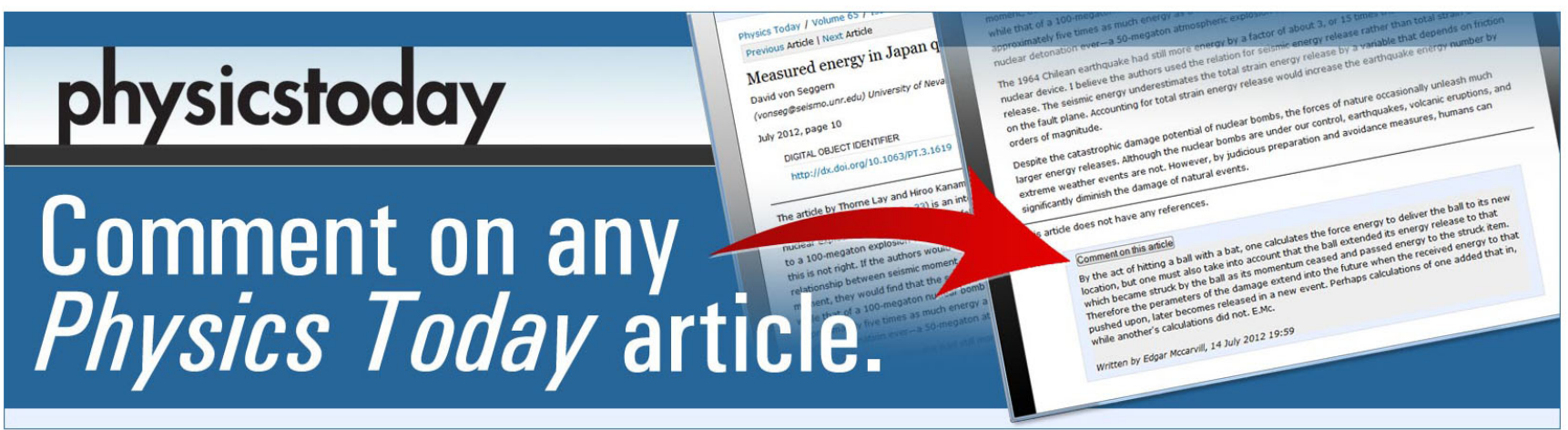




\title{
Polarizer design for millimeter-wave plasma diagnostics
}

\author{
F. Leipold, ${ }^{\text {a) }}$ M. Salewski, A. S. Jacobsen, M. Jessen, S. B. Korsholm, P. K. Michelsen, \\ S. K. Nielsen, and M. Stejner \\ Department of Physics, Technical University of Denmark, DK-4000 Roskilde, Denmark
}

(Received 3 May 2013; accepted 12 July 2013; published online 1 August 2013)

\begin{abstract}
Radiation from magnetized plasmas is in general elliptically polarized. In order to convert the elliptical polarization to linear polarization, mirrors with grooved surfaces are currently employed in our collective Thomson scattering diagnostic at ASDEX Upgrade. If these mirrors can be substituted by birefringent windows, the microwave receivers can be designed to be more compact at lower cost. Sapphire windows (a-cut) as well as grooved high density polyethylene windows can serve this purpose. The sapphire window can be designed such that the calculated transmission of the wave energy is better than $99 \%$, and that of the high density polyethylene can be better than $97 \%$. ( 2013 AIP Publishing LLC. [http://dx.doi.org/10.1063/1.4816724]
\end{abstract}

\section{INTRODUCTION}

Electromagnetic radiation penetrating into or escaping from a magnetized plasma is generally elliptically polarized. Since sources and receivers are usually capable of sending and receiving linearly polarized waves only, a conversion from linearly to elliptically polarized waves or vice versa is then usually performed. This conversion can be done by any means that changes the phase between two perpendicular electric fields. We focus here on changing the polarization of millimeter waves.

The authors in Ref. 1 used metal plate arrays for this purpose in an electron cyclotron emission (ECE) radiometer. While the electric field component perpendicular to the metal plates is not affected by the plate array, the parallel field component propagates at higher phase velocity. The phase between perpendicular and parallel electric component can be adjusted by varying the length of the metal plate array in propagation direction and the distance between individual metal plates.

Interferometers operating in the $\mathrm{mm}$ wavelength range use wire grids and roof top mirrors to manipulate phase and orientation of the electric field components. ${ }^{2,3}$ Wire grids reflect the electric field component parallel to the wires while the component perpendicular to the wires remains unaffected. The phase between the two separated components can be manipulated by changing the optical path length of the waves. Roof top mirrors consist of two perpendicularly oriented flat mirrors. The intersecting line between the mirrors is perpendicular to the direction of propagation of the incident and reflected wave. The orientation of the reflected electric field component depends on the orientation of the intersecting line of the mirrors and the orientation of the incident electric field component. For details see Ref. 3.

For high power applications such as electron cyclotron resonance heating $(\mathrm{ECRH})$ or electron cyclotron current drive (ECCD), the conversion from linearly to elliptically polarized waves is performed using grooved metal mirrors. ${ }^{4-6}$ In a simplified picture, the electric field component parallel to the

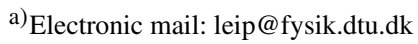

grooves is reflected off the top of the grooves, and the electric field component perpendicular to the grooves is reflected off the bottom of the grooves. This causes a phase shift between the two components that depends on the depth of the grooves. The advantages of using metal mirrors are that high power can be transmitted and that the losses are low. The disadvantage is the large required space. These types of mirrors are also currently employed in the quasi-optical transmission line of our collective Thomson scattering (CTS) diagnostic at ASDEX Upgrade..$^{7-12}$ The spatial dimension of the quasioptical transmission could be reduced, if a birefringent window can be inserted into the transmission line instead of grooved mirrors. This technique is of particular interest for the CTS diagnostic in ITER. ${ }^{13-15}$ The CTS system in ITER is supposed to have ten spatial CTS channels, requiring ten sets of polarizer plates. Space limitations probably will not allow the installation of that many polarizer mirrors. One option is to ignore the polarization and to accept the reduced intensity by detecting only one linear component of the elliptically polarized beam. The other option is to employ sapphire windows with a defined orientation just in front of the horn antenna to perform the conversion to linearly polarized waves. Windows would also make the system less vulnerable to misalignment in comparison with mirrors. However, windows have the disadvantage that the transmission is never completely without losses, even if the material is completely ideal and loss free: There is always some reflection on the surface between two media with different refractive indices. Interference between multiple waves reflected from the front and back surfaces of the window can cause a substantial change of the effective transmission within a small wavelength change. This interference pattern is superimposed to the spectral intensity profile and may be too severe to be ignored. We investigate two types of materials suitable for windows for our CTS receiver: (1) grooved high density polyethylene (HDPE) plates, and (2) sapphire windows.

Such birefringent windows are also applicable to other low power microwave systems such as ECE or correlated ECE detection, ${ }^{16-18}$ polarimetry, reflectometry ${ }^{19}$ or Doppler back scattering, where probing and receiving beams penetrate or leave the plasma under an oblique angle. ${ }^{20-22}$ 
In Sec. II, we give a short summary of the theoretical background of polarizer windows. In Sec. III, we describe the experimental setup for the characterization of the birefringent windows. Section IV presents the measurement as well as a theoretical characterisation of the transmission. In Sec. V, we evaluate the applicability of the described polarizers for our diagnostic, and conclusions are drawn in Sec. VI.

\section{THEORETICAL BACKGROUND}

The dielectric susceptibility of an anisotropic medium can in general be described by a tensor. As the tensor is symmetric, one can always choose the axes of the coordinate system such that the dielectric susceptibility tensor is diagonal, i.e., the off-diagonal elements are zero. The coordinate axes are then the principal axes of the medium, which we choose as local coordinate axes of the medium from here on. The electric field component in $\mathrm{x}$-direction, $\mathrm{E}_{x}$, sees the refractive index $\mathrm{n}_{e}$, while the electric field component in $\mathrm{y}$-direction, $\mathrm{E}_{y}$, sees the refractive index $\mathrm{n}_{o}$ (Fig. 1). The indices e and o refer to the commonly used terms extraordinary and ordinary modes, respectively. These should not be confused with ordinary and extraordinary modes of the millimeter wave beam in the plasma. The change of the phase shift, $\psi$, between $\mathrm{E}_{x}$ and $\mathrm{E}_{y}$ after transmission through an anisotropic medium is given by

$$
\psi=2 \pi \frac{d}{\lambda} \times\left(n_{e}-n_{o}\right),
$$

where $\mathrm{d}$ is the thickness of the medium and $\lambda$ is the wavelength in vacuum. Mathematically, the relation between the incident electric field, $\mathbf{E}_{i n}$, and the transmitted electric field, $\mathbf{E}_{\text {out }}$, is given by the following equation:

$$
\mathbf{E}_{\text {out }}=\left(\begin{array}{cc}
e^{i \psi} & 0 \\
0 & 1
\end{array}\right) \mathbf{E}_{\text {in }} .
$$

If the medium is rotated around the z-axis by the angle $\phi$, it is convenient to transform the incident electric field, $\mathbf{E}_{i n}$, to a local coordinate system given by the principal axes of the media (Fig. 1). After transmission through the media, it is convenient to transform the electric field back into the laboratory coordinate system as the detector is aligned with this system. The electric field, $\mathbf{E}_{\text {out }}$, seen by the detector is mathe-

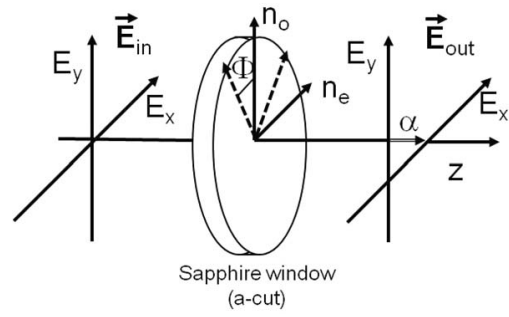

FIG. 1. Sapphire is a birefringent material and can be used for polarization purposes. The inherent optical axis lies within the window plane (a-cut). $\alpha$ symbolizes the phase shift between $\mathrm{E}_{x}$ and $\mathrm{E}_{y}$ of $\mathbf{E}_{\text {out }} . \alpha=\psi$, if $\phi=0$ and the electric field components $\mathrm{E}_{x}$ and $\mathrm{E}_{y}$ of $\mathbf{E}_{i n}$ are in phase. The dashed arrows depict the principal axes and the local coordinate system of the window if it is rotated around the $\mathrm{z}$-axis by the angle $\Phi$.

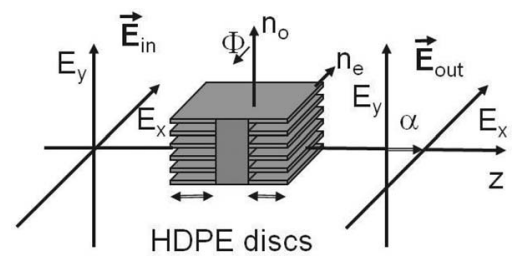

FIG. 2. Staggered HDPE plates can serve as birefringent window and be used for polarization purposes. $\alpha$ symbolizes the phase shift between $\mathrm{E}_{x}$ and $\mathrm{E}_{y}$ of $\mathbf{E}_{\text {out }}$. The bi-directional arrows indicate the grooved section of the material. The center section between the bi-directional arrows does not contribute to polarization effects.

matically described by

$$
\mathbf{E}_{\text {out }}=\mathbf{R}\left(\begin{array}{cc}
e^{i \psi} & 0 \\
0 & 1
\end{array}\right) \mathbf{R}^{\prime} \mathbf{E}_{\text {in }}
$$

where the rotation matrix, $\mathbf{R}$, is given by

$$
\mathbf{R}=\left(\begin{array}{cc}
\cos (\phi) & -\sin (\phi) \\
\sin (\phi) & \cos (\phi)
\end{array}\right) .
$$

Single crystal sapphire can be used as a birefringent material if two principal axes lie in the window plane, and one of them has to be the inherent optical axis of the crystal (a-cut). The authors in Ref. 23 used the birefringent properties of sapphire to build achromatic half-wave plates. An isotropic material such as HDPE can also be used as an effectively anisotropic medium by staggering HDPE plates on top of each other with gaps in between (Fig. 2). It serves the same purpose as a birefringent crystal. Practically, grooves were milled in both sides of an HDPE window. ${ }^{24}$ The center part of the HDPE window does not contribute to a phase shift between $\mathrm{E}_{x}$ and $\mathrm{E}_{y}$. Only the grooved sections do. It would be possible to mill grooves in only one side, but from a machining point of view it is easier to machine both sides and make the grooves less deep. Figure 3 shows photographs of the HDPE and the sapphire windows used in this work. These two types of birefringent windows shall be used in order to
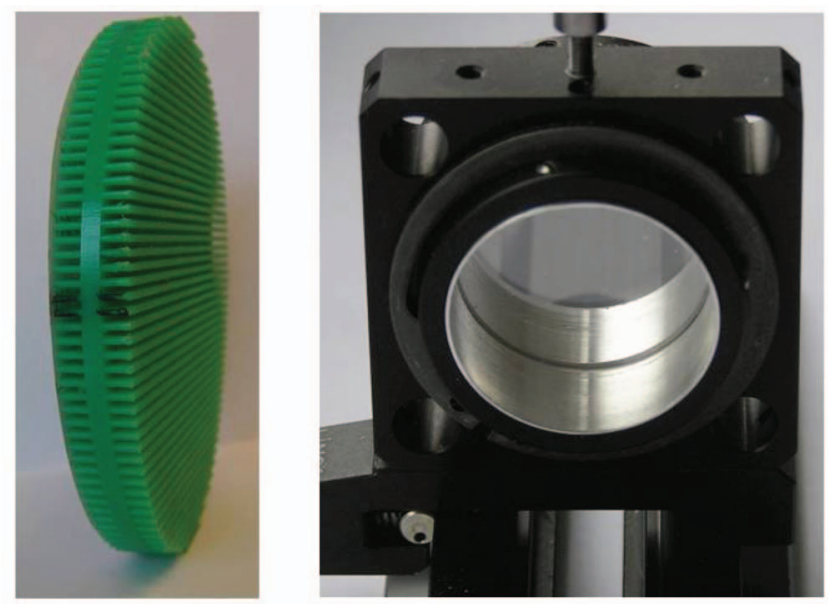

FIG. 3. Photos of the grooved HDPE window (left) and the sapphire window with anti-reflection coatings mounted in an optical holder (right). The diameter of the HDPE window is $90 \mathrm{~mm}$, and the diameter of the sapphire window is $25 \mathrm{~mm}$. 


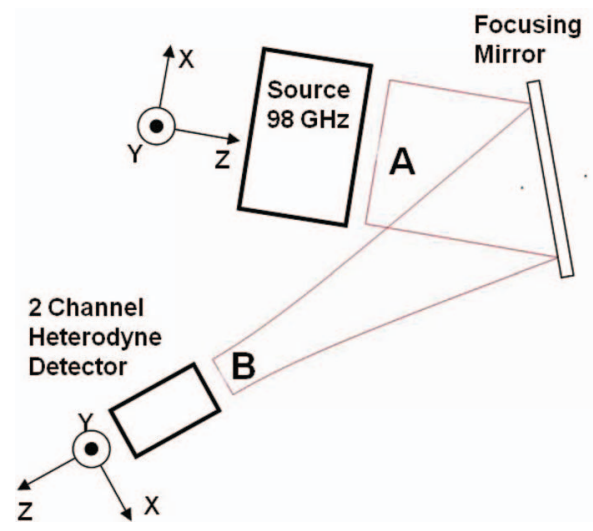

FIG. 4. A linearly polarized (x-direction) microwave beam $(98 \mathrm{GHz})$ with a beam waist of $28 \mathrm{~mm}$ was focussed by means of a focusing mirror to a beam waist of $7.8 \mathrm{~mm}$. A two-channel heterodyne detector was used to measure the electric field components $\mathrm{E}_{x}$ and $\mathrm{E}_{y}$ and the phase shift, $\alpha$, in between. Test windows can be placed at $\mathrm{A}$ and $\mathrm{B}$ and rotated around the $\mathrm{z}$-axis.

convert elliptically polarized scattered waves into linearly polarized waves detectable for our receiver.

\section{EXPERIMENTAL SETUP}

The experimental setup is shown in Fig. 4. The microwave source $(98 \mathrm{GHz})$ emits linearly polarized waves with the electric field vector pointing in $\mathrm{x}$-direction. The Gaussian beam radius at the exit port of the source is $28 \mathrm{~mm}$. This is also the beam waist. The beam is reflected by means of a focusing mirror. The profile of the mirror is made such that the reflected beam has a beam waist at location B in Fig. 4. The Gaussian beam radius is $7.8 \mathrm{~mm}$ at the beam waist. The dimensions are chosen such that the source has the same beam characteristic as it is expected from the waveguide at ASDEX Upgrade, and the receiver side sees the same beam dimension as required for the horn antenna in our CTS receiver at ASDEX Upgrade. The radiation is detected by means of a two-channel heterodyne receiver, which detects $\mathrm{E}_{x}$ and $\mathrm{E}_{y}$ components of the electric field separately and measures the phase shift, $\alpha$, between $\mathrm{E}_{x}$ and $\mathrm{E}_{y}$. The two-channel heterodyne receiver is detailed in Ref. 25. The birefringent windows can be inserted at positions A and B in Fig. 4, and they can be rotated around the z-axis similarly to their envisioned use in the CTS receiver at ASDEX Upgrade. The measurements at various rotation angles $\phi$ are obtained with the twochannel heterodyne detector and are compared with calculations obtained by using Eq. (3). It is important to find a model that can describe the polarization for various rotation angles of the window, for example, to calculate the polarizer settings in CTS experiments. As will be seen in Sec. IV, Eq. (3) describes the measured polarization in good approximation. This ensures that the expected scattered radiation is converted to linearly polarized waves to be detected by the receiver. The change of direction of polarization for a window inserted in position A due to reflection at the focusing mirror has to be taken into account (Fig. 4). Both types of windows, sapphire and HDPE, were designed as $\lambda / 4$ plates for $105 \mathrm{GHz}$. The required thickness of the sapphire window in order to generate a phase shift of $\psi=\pi / 2$ was calculated (Eq. (1)) using the
TABLE I. Refractive indices of sapphire, suprasil, and HDPE at room temperature. Here, $\tan (\delta)$ is the ratio of the imaginary and the real part of the dielectric susceptibility.

\begin{tabular}{llcccc}
\hline \hline & & $\begin{array}{c}\text { Refractive } \\
\text { index }\end{array}$ & $\begin{array}{c}\tan (\delta) \\
{\left[10^{-4}\right]}\end{array}$ & $\begin{array}{c}\text { Frequency } \\
{[\mathrm{GHz}]}\end{array}$ & $\begin{array}{c}\text { Source } \\
\text { (Refs.) }\end{array}$ \\
\hline Sapphire & $\mathrm{n}_{o}$ & $3.066-3.071$ & $2.1-2.5$ & 140 & 26 \\
& $\mathrm{n}_{e}$ & $3.400-3.405$ & $1.1-1.4$ & & 26 \\
Suprasil & $\mathrm{n}$ & 1.81 & 1.7 & & 27 \\
HDPE & $\mathrm{n}$ & 1.53 & 3.1 & 160 & 27 \\
& $\mathrm{n}_{o}$ & 1.18 & & & 24,27 \\
& $\mathrm{n}_{e}$ & 1.28 & & & 24,27 \\
\hline \hline
\end{tabular}

refractive indices $\mathrm{n}_{o}$ and $\mathrm{n}_{e}$ provided in Ref. 26 and listed in Table I. We use the values in Table I for our application at a frequency of $105 \mathrm{GHz}$, while the listed values in Table I are given for $140 \mathrm{GHz}$, and therefore the refractive index values may be inaccurate for $105 \mathrm{GHz}$. Any inaccuracy in refractive index will change the measured phase shift according to Eq. (1), but the conversion from elliptical to linear polarization will still be possible, if just a different thickness of the window is used (Eq. (1)). The required thickness was calculated to be $2.1 \mathrm{~mm}$. Due to the lack of a source radiating at $105 \mathrm{GHz}$, a $98 \mathrm{GHz}$ source was used instead. This affects the phase shift between $\mathrm{E}_{x}$ and $\mathrm{E}_{y}$, but again the results can be corrected using Eq. (1).

The required thickness of the grooved sections of the HDPE windows in order to generate a phase shift of $\pi / 2$ between the electric field vector perpendicular and parallel to the grooves was calculated using Eq. (1) and the refractive indices $\mathrm{n}_{o}$ and $\mathrm{n}_{e}$ given in Table I. $\mathrm{n}_{o}$ and $\mathrm{n}_{e}$ were calculated using the refractive index, $\mathrm{n}$, for HDPE and the formulas provided in Ref. 24 (Table I). The required total thickness of both grooved sections of the HDPE window together was calculated to be $6.7 \mathrm{~mm}$. The period of the fins of the HDPE plate is $2 \mathrm{~mm}$ and the aspect ratio is 0.5 . These dimensions meet the requirements given in Ref. 24, where the thickness of the fins and the width of the grooves shall be approximately $\lambda / 3$ or less.

\section{RESULTS}

\section{A. Sapphire window}

The sapphire window was placed at position B in Fig. 4. Figure 5 shows measurements of the electric field in $\mathrm{x}$ - and $\mathrm{y}$ directions for each $10^{\circ}$ of rotation of the window around the $\mathrm{z}$-axis. The symbols represent the measurement, and the solid lines represent the calculation using Eq. (3). The phase shift between $\mathrm{E}_{x}$ and $\mathrm{E}_{y}$ in the calculation was varied to obtain the best fit to experimental data. The best fit between measurements and calculations was found for $\psi=77.5^{\circ}$ in the calculation. The rather big difference to the intended phase shift of $90^{\circ}$ is attributed to the uncertainty of the refractive indices $\mathrm{n}_{o}$ and $\mathrm{n}_{e}$. In order to calculate the thickness of the sapphire window for a given phase shift with an accuracy better than $1^{\circ}$, the refractive indices must be known with an accuracy better than $0.1 \%$. These measurements can be used to recalculate the thickness of the sapphire window according to Eq. (1). 

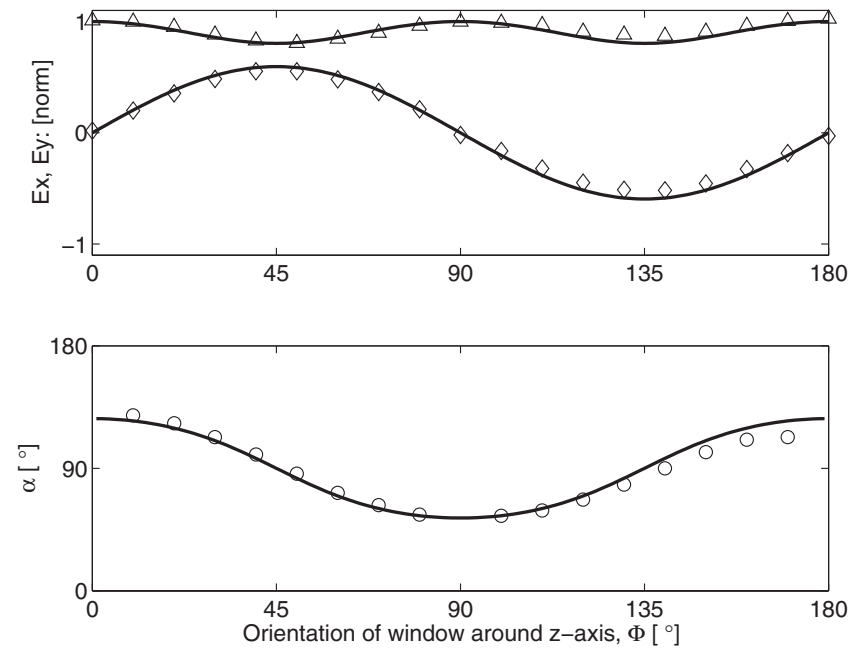

FIG. 5. Measurement of the electric field $\mathrm{E}_{x}$ (triangles), $\mathrm{E}_{y}$ (diamonds), and the phase, $\alpha$, between $\mathrm{E}_{x}$ and $\mathrm{E}_{y}$ (circles). The symbols represent the measurements, and the solid lines show the calculation. The best fit between measurements and calculations was found for $\psi=77.5^{\circ}$ in the calculation.

\section{B. High density polyethylene window}

The HDPE window was placed at position A in Fig. 4. Figure 6 shows measurements of the electric field in $\mathrm{x}$ - and $\mathrm{y}$ directions for each $15^{\circ}$ of rotation of the window around the $\mathrm{z}$-axis and the corresponding calculation. The symbols represent the measurement, and the solid lines represent the calculations using Eq. (3). The phase shift of the computation was again varied to obtain the best fit to the measurements. The best fit between measurements and calculations was found for $\psi=87^{\circ}$ in the calculation.

\section{Transmission through the sapphire window}

The reflection of radiation at the surface of a sapphire window at a frequency on the order of $100 \mathrm{GHz}$ is quite substantial. This is attributed to the high refractive index of sap-
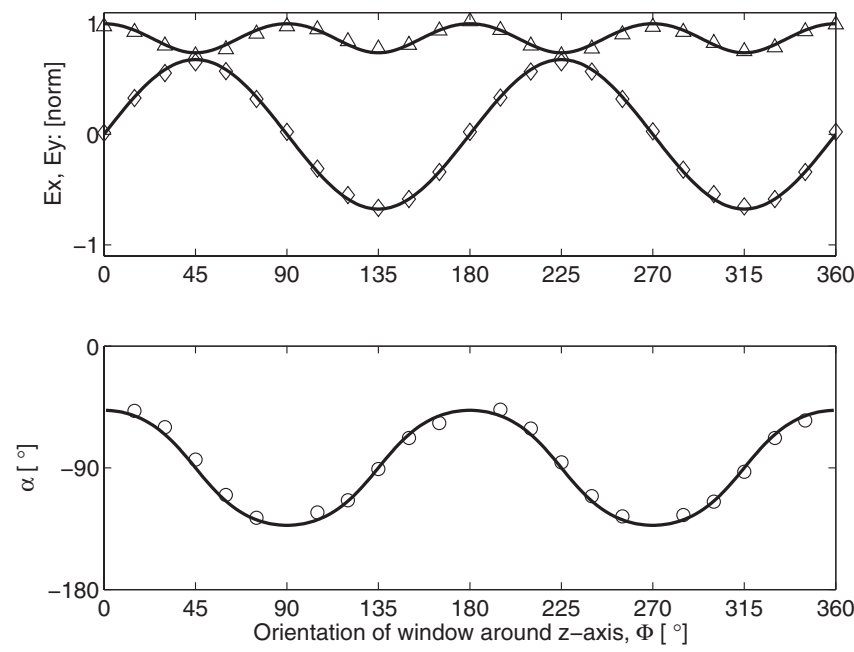

FIG. 6. Measurement of the electric field $\mathrm{E}_{x}$ (triangles), $\mathrm{E}_{y}$ (diamonds), and the phase, $\alpha$, between $\mathrm{E}_{x}$ and $\mathrm{E}_{y}$ (circles). The symbols represent the measurements, and the solid lines show the calculation. The best fit between measurements and calculations was found for $\psi=87^{\circ}$ in the calculation.

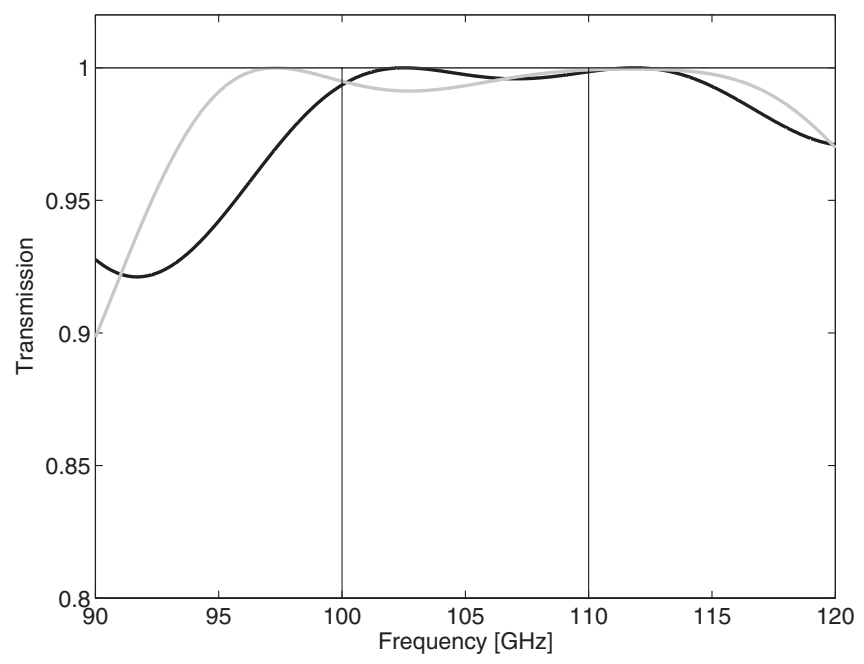

FIG. 7. Transmission of the sapphire window for o-mode (black) and $\mathrm{x}$-mode (grey) with suprasil windows as anti-reflection layer. The transmission in the wavelength range of interest $(100-110 \mathrm{GHz})$ is calculated to be better than 99\%. Losses in the sapphire are not considered in this calculation.

phire (Table I). Approximately $25 \%$ of the radiation power is reflected. In order to improve the transmission, two suprasil windows with a refractive index of 1.8 (Table I) and a thickness of $0.38 \mathrm{~mm}$ each were placed in contact with each surface of the sapphire window. The calculated transmission for such a three window assembly for o- and $\mathrm{x}$-mode is shown in Fig. 7. The transmission within our region of interest $(100 \mathrm{GHz}-110 \mathrm{GHz})$ is better than $99 \%$. Losses in the sapphire window were not considered in this calculation. The power absorption in the sapphire window can be calculated using $\tan (\delta)$ given in Table I. The power absorption for the window with a thickness of $2.1 \mathrm{~mm}$ was calculated to be at most $0.35 \%$ and $0.22 \%$ in the o- and $\mathrm{x}$-mode, respectively, using the values given in Table I.

\section{Transmission through the HDPE window}

In order to determine the transmission of the grooved HDPE window, we used a similar model as for the suprasilsapphire-suprasil assembly. The grooved sections on each side of the HDPE window were considered as one layer each, and the center part was considered as a third layer. The effective refractive indices $\mathrm{n}_{o}$ and $\mathrm{n}_{e}$ for the grooved section and the refractive index for HDPE for the center section are given in Table I. Figure 8 shows the transmission for linearly polarized waves in o-mode and $x$-mode. An HDPE $\lambda / 4$ window shows considerable losses of up to $15 \%$. If the thickness of the grooved sections and of the center part of the window are changed, the transmission can be better than 97\% (Fig. 9). The drawback is that this HDPE window is not a $\lambda / 4$ window at $105 \mathrm{GHz}$ any more. The phase shift between the ordinary and the extraordinary mode is $100^{\circ}$. However, such a window (or a set of two of them) can still serve the purpose of converting elliptically polarized waves to linearly polarized waves. 


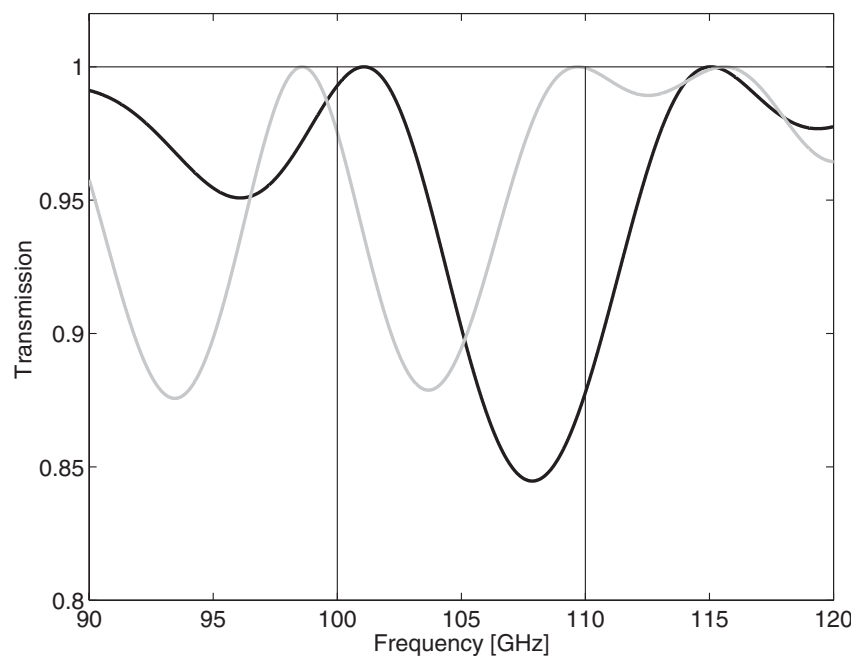

FIG. 8. Calculation of the transmission of the wave energy of the o-mode (black) and $\mathrm{x}$-mode (grey), when the HDPE window is designed as a $\lambda / 4$ plate. Losses in the HDPE are not considered in this calculation.

\section{DISCUSSION}

Sapphire windows (a-cut) as well as grooved HDPE windows can be used for conversion of elliptically polarized light into linearly polarized light. The rather large discrepancy between the designed phase shift $\left(90^{\circ}\right)$ of the sapphire window and the measured phase shift $\left(77.5^{\circ}\right)$ can be due to reflections most probably caused by the cylindrical tube in which the sapphire window is mounted. Such reflection can alter the original linearly polarized beam to become slightly elliptic. The aperture of this tube is 1.47 times the Gaussian width of the wave at that position. In order to overcome this problem, the cylindrical tube can be designed shorter and the window can be made larger in diameter. The size of the beam is an important factor for the choice whether a sapphire or a grooved HDPE window should be used. If the beam diameter is large, a grooved HDPE window is preferable, since it is difficult and

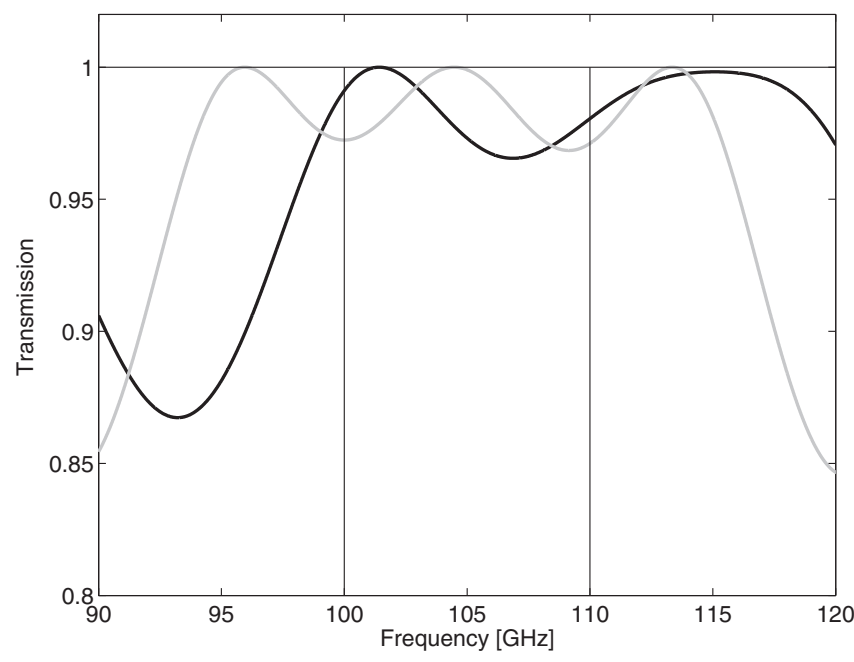

FIG. 9. Calculation of the transmission of the wave energy of the omode (black) and x-mode (grey), when the HDPE window is designed for $\psi=100^{\circ}$ between the ordinary and extraordinary mode. Losses in the HDPE are not considered in this calculation. expensive to make a large, thin sapphire window and even more difficult to make the required anti-reflection windows. If the beam diameter is small, the sapphire window is preferable since the grooved HDPE window is fairly thick and the beam can diverge significantly within a thick window. However, in radiative environment such as in ITER or future reactors, HDPE windows would degrade quickly, while sapphire windows are still a good option despite the large size of the beam. For grooved HDPE windows, the phase shift cannot be chosen freely. The dimensions of the grooved sections and the center part have to be optimized for low reflections. However, this is not a drawback, since the orientation for a required polarization can be calculated. Generally, an anti-reflection layer can also be considered for HDPE windows. They would have a similar thickness as the anti-reflection windows for the sapphire windows. However, this is difficult to employ since HDPE is relatively flexible and not necessarily very plane. In this case, gaps between the anti-reflection window may form, rendering the effect of an anti-reflection window worthless. Therefore, anti-reflection windows for HDPE windows seem not very practical. However, different techniques reducing reflections at the surface of grooved HDPE windows can be considered. The rectangular fins may have triangular transitions at both ends or they can be made entirely triangular instead or rectangular. ${ }^{28,29}$ This provides a gradual transition from the refractive index of air to the refractive index of HDPE. The aspect ratio of the grooves can be optimized such that the thickness of the grooved section is $(2 n+1) \times \lambda / 4$. This would form an anti-reflection layer. However, if $n$ is large the bandwidth is small and may not cover the required frequency range. In addition, the thickness of the solid part of the window can be chosen such that it becomes resonant for the center frequency. The authors in Ref. 30 used a $\lambda / 4$ quartz window in a polarimetry diagnostic in order to manipulate the polarization properties of the probing beam. The applicability of cooled sapphire vacuum windows for ECRH has been investigated in Ref. 31, but replacement of polarizer mirrors by sapphire windows for ECRH has not been reported.

\section{CONCLUSION}

Sapphire windows with the crystal axis in the window plane (a-cut) as well as grooved HDPE windows can be used for conversion of elliptically polarized light into linearly polarized light or vice versa. The replacement of grooved mirrors by birefringent windows simplifies the quasi-optics and is principally applicable to other low power microwave systems such as ECE or correlated ECE detection, ${ }^{16-18}$ polarimetry, reflectometry ${ }^{19}$ or Doppler back scattering, where probing and receiving beam penetrates or leaves the plasma under an oblique angle. ${ }^{20-22}$ While a power absorption of less than $1 \%$ in sapphire is still too large for high power application such as ECRH or ECCD, it is easily acceptable for low power millimeter wave diagnostics.

${ }^{1}$ F. Volpe, M. E. Austin, G. Campbell, and T. Deterly, Rev. Sci. Instrum. 83, 103507 (2012).

${ }^{2}$ M. Kotiranta, C. Leinz, T. Klein, V. Krozer, and H. J. Wunsch, J. Infrared Millim. Terahertz Waves 33, 1138 (2012). 
${ }^{3}$ S. Schmuck, J. Fessey, T. Gerbaud, B. Alper, M. N. A. Beurskens, E. de la Luna, A. Sirinelli, M. Zerbini, and J.-E. Contributorsb, Rev. Sci. Instrum. 83, 125101 (2012).

${ }^{4}$ D. Wagner and F. Leuterer, Int. J. Infrared Millim. Waves 26, 163 (2005).

${ }^{5}$ D. Wagner, F. Leuterer, A. Manini, F. Monaco, M. Munich, F. Ryter, H. Schuetz, H. Zohm, T. Franke, R. Heidinger et al., Int. J. Infrared Millim. Waves 27, 173 (2006).

${ }^{6}$ M. K. Thumm and W. Kasparek, IEEE Trans. Plasma Sci. 30, 755 (2002).

${ }^{7}$ F. Meo, H. Bindslev, S. B. Korsholm, V. Furtula, F. Leuterer, F. Leipold, P. K. Michelsen, S. K. Nielsen, M. Salewski, J. Stober et al., Rev. Sci. Instrum. 79, 10E501 (2008).

${ }^{8}$ F. Meo, M. Stejner, M. Salewski, H. Bindslev, T. Eich, V. Furtula, S. B. Korsholm, F. Leuterer, F. Leipold, P. K. Michelsen et al., J. Phys.: Conf. Ser. 227, 012010 (2010).

${ }^{9}$ M. Salewski, F. Meo, M. Stejner, O. Asunta, H. Bindslev, V. Furtula, S. B. Korsholm, T. Kurki-Suonio, F. Leipold, F. Leuterer et al., Nucl. Fusion 50, 035012 (2010)

${ }^{10}$ V. Furtula, F. Leipold, M. Salewski, P. K. Michelsen, S. B. Korsholm, F Meo, D. Moseev, S. K. Nielsen, M. Stejner, and T. Johansen, J. Instrum. 7, C02039 (2012).

${ }^{11}$ V. Furtula, M. Salewski, F. Leipold, P. K. Michelsen, S. B. Korsholm, F. Meo, D. Moseev, S. K. Nielsen, M. Stejner, and T. Johansen, Rev. Sci. Instrum. 83, 013507 (2012).

${ }^{12}$ M. Salewski, B. Geiger, S. Nielsen, H. Bindslev, M. García-Muñoz, W. Heidbrink, S. Korsholm, F. Leipold, F. Meo, P. Michelsen et al., Nucl. Fusion 52, 103008 (2012).

${ }^{13}$ M. Salewski, F. Meo, H. Bindslev, V. Furtula, S. B. Korsholm, B. Lauritzen, F. Leipold, P. K. Michelsen, S. K. Nielsen, and E. Nonbøl, Rev. Sci. Instrum. 79, 10E729 (2008)

${ }^{14}$ F. Leipold, V. Furtula, M. Salewski, H. Bindslev, S. Korsholm, F. Meo, P. Michelsen, D. Moseev, S. Nielsen, and M. Stejner, Rev. Sci. Instrum. 80, 093501 (2009).
${ }^{15}$ M. Salewski, O. Asunta, L.-G. Eriksson, H. Bindslev, V. Hynönen, S. B. Korsholm, T. Kurki-Suonio, F. Leipold, F. Meo, P. K. Michelsen et al., Plasma Phys. Controlled Fusion 51, 035006 (2009).

${ }^{16}$ L. Schmitz, A. E. White, G. Wang, J. C. DeBoo, J. S. deGrassie, G. R. McKee, J. C. Hillesheim, W. A. Peebles, T. L. Rhodes, T. A. Carter et al., Nucl. Fusion 49, 095004 (2009).

${ }^{17}$ A. E. White, W. A. Peebles, T. L. Rhodes, C. H. Holland, G. Wang, L. Schmitz, T. A. Carter, J. C. Hillesheim, E. J. Doyle, L. Zeng et al., Phys. Plasmas 17, 056103 (2010).

${ }^{18}$ J. C. Hillesheim, W. A. Peebles, T. L. Rhodes, L. Schmitz, A. E. White, and T. A. Carter, Rev. Sci. Instrum. 81, 10D907 (2010).

${ }^{19}$ H. J. Hartfuss, T. Geist, and M. Hirsch, Plasma Phys. Controlled Fusion 39, 1693 (1997)

${ }^{20}$ E. Holzhauer, M. Hirsch, T. Grossmann, B. Branas, and F. Serra, Plasma Phys. Controlled Fusion 40, 1869 (1998).

${ }^{21}$ M. Hirsch, E. Holzhauer, J. Baldzuhn, B. Kurzan, and B. Scott, Plasma Phys. Controlled Fusion 43, 1641 (2001).

${ }^{22}$ J. C. Hillesheim, W. A. Peebles, T. L. Rhodes, L. Schmitz, T. A. Carter, P. A. Gourdain, and G. Wang, Rev. Sci. Instrum. 80, 083507 (2009).

${ }^{23}$ S. Hanany, J. Hubmayr, B. R. Johnson, T. Matsumura, P. Oxley, and M. Thibodeau, Appl. Opt. 44, 4666 (2005).

${ }^{24}$ A. H. F. van Vliet and T. de Graauw, Int. J. Infrared Millim. Waves 2, 465 (1981).

${ }^{25}$ F. Leipold, S. K. Nielsen, and S. Michelsen, Rev. Sci. Instrum. 79, 065103 (2008).

${ }^{26}$ V. V. Parshin, Int. J. Infrared Millim. Waves 15, 339 (1994).

${ }^{27}$ J. W. Lamb, Int. J. Infrared Millim. Waves 17, 1997 (1996).

${ }^{28}$ J. Y. L. Ma and L. C. Robinson, Opt. Acta 30, 1685 (1983).

${ }^{29}$ M. Petelin and W. Kasparek, Int. J. Electron. 71, 871 (1991).

30 J. Zhang, W. A. Peebles, T. A. Carter, N. A. Crocker, E. J. Doyle, S. Kubota, X. Nguyen, T. L. Rhodes, C. Wannberg, and L. Zeng, Rev. Sci. Instrum. 83, 10E321 (2012)

${ }^{31}$ M. Thumm and W. Kasparek, Fusion Eng. Des. 26, 291 (1995). 